\title{
Next steps in preventing Ewing sarcoma progression
}
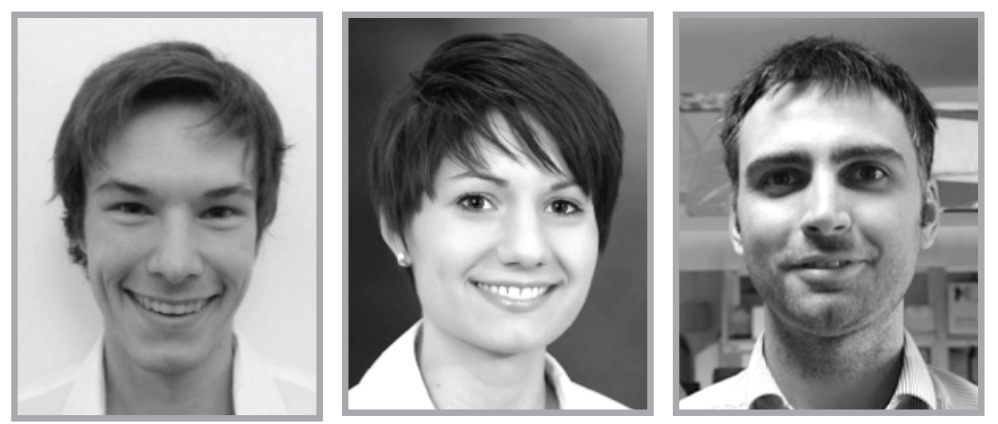

Max ML Knott ${ }^{\ddagger 1}$, Marlene Dallmayer ${ }^{\ddagger 1,}$ \& Thomas GP Grünewald ${ }^{*, 1}$

First draft submitted: 21 October 2015; Accepted for publication: 22 October 2015; Published online: 30 November 2015

Ewing sarcoma (EwS) is a highly aggressive malignant tumor of bone or soft tissue, mostly affecting adolescents and young adults [1]. Metastasis is a catastrophic event in the clinical course of EwS patients [1]. Although prevention of metastasis appears key for improving outcome, no specific therapeutic strategies have yet been implemented in routine clinical protocols specifically blocking EwS progression. Here, we summarize the current knowledge on several aspects that we consider being essential for a better biological understanding of EwS progression, and propose next steps for future research and therapeutic intervention.

\section{Contribution of germline variability}

We propose that EwS may constitute a genuine model to study the complex ways of how the interplay of germline variation and somatic mutation might promote tumor progression [2]:

- EwS features a relatively simple genetic architecture with a nearly diploid genome, a single somatic driver mutation (such as the chimeric transcription factor EWSR1FLI1) [3] and only a few germline susceptibility loci with strong effect sizes [2,4];

- EWSR1-FLI1 hijacks GGAA-microsatellites (mSats) as enhancers to drive the expression of clinically relevant oncogenes [5]. As GGAA-mSats underlie strong germline variability, this feature provides the genetic basis to explore how germline variability in interaction with a somatic driver mutation (EWSR1-FLI1) may translate into tumor heterogeneity and differential patient outcome [2].

EWSR1-FLI1 acts as a pioneer transcription factor converting GGAA-mSats into active enhancers $[5,6]$. Binding of EWSR1FLI1 to GGAA-mSats causes the chromatin to open at these otherwise nonfunctional 'junk' DNA elements [5,6], which enables EWSR1-FLI1 to steer many of its target genes. Some of them, such as NROB1 (alias DAX1), GSTM4 and EGR2, are critically involved in tumorigenesis, tumor

\section{KEYWORDS}

- angiogenesis $\bullet$ Ewing sarcoma

- EWSR1-FLI1 • immunotherapy

- metastasis

'Laboratory for Pediatric Sarcoma Biology, Institute of Pathology of the LMU Munich, Munich, Germany

*Author for correspondence: Tel.: +49 892180 73716; Fax: +49 892180 73604;

thomas.gruenewald@med.uni-muenchen.de

${ }^{\ddagger}$ Authors contributed equally

Future
Medicine
part of 
"We foresee that immunological approaches based on adoptive transfer of NK or T cells will enter routine clinical practice in the near future." progression and/or drug resistance of EwS [4,7-8]. Kwon et al. proposed that each GGAA-repeat within a given GGAA-mSat may be occupied by a FLI1 domain of EWSR1-FLI1, thereby clustering multiple EWSR1-FLI1 proteins in close proximity that the low-complexity domains of EWSR1 start polymerizing [9]. This creates a polymeric docking site for RNA polymerase II [9], which would at least partially explain why the number of GGAArepeats correlates with the enhancer activity of EWSR1-FLI1-bound GGAA-mSats [5].

However, the enhancer activity conferred by EWSR1-FLI1 shows - as exemplified at the NROB1-associated GGAA-mSat - a bimodal distribution $[5,10]$. Also, the enhancer activity appears to increase exponentially above a certain threshold and minor interruptions of GGAA$m$ Sats can cause dramatic loss of enhancer activity for unknown reasons $[4-5,10]$.

Yet, major questions remain largely unexplored: Why does EWSR1-FLI1 first start binding to GGAA-mSats with a minimum of four repeats? Why is there an exponential increase of enhancer activity above 12-13 GGAA-repeats? Why is there a bimodal distribution of enhancer activity above 24 GGAA-repeats? Why are some interruptions of active GGAA-mSats well tolerated, while others abolish enhancer activity? Which patterns of GGAA-mSats are preferred in a bi-allelic configuration when considering the diploid nature of the human genome?

As specific genes driven through the oncogenic interplay of EWSR1-FLI1 and GGAA-mSats may cause the clinically observed heterogeneity in patient outcome, we propose that clarification of these questions may be key for our understanding of how these genes are activated and how to apply this knowledge to prevent tumor progression.

\section{Adaptation to cell stress}

Cytotoxic drugs, irradiation and the tumor microenvironment with its specific nutritional and hypoxic conditions constitute sources of cellular stress. However, the ways in which EwS cells adapt to cellular stress and whether this promotes tumor progression is poorly understood.

Recent evidence shows that under conditions of cell stress, EwS cells utilize the conserved RNAbinding protein YBX1 and the stress-granule nucleator G3BP1 to transiently reduce energy consumption by downregulation of global protein translation through sequestration of mRNA templates in stress granules [11]. However, stressmediated activation of YBX1 induces specific translation of HIF1 $\alpha$, which in turn promotes EwS cell adaptation to hypoxia, resulting in higher metastatic potential [12]. High YBX1 or G3BP1 expression are consistently associated with worse clinical outcome [11,12]. Krook et al. provided further evidence for the important role of cellular stress in EwS progression [13]. The chemokine receptor, CXCR4, is dynamically upregulated under cellular stress conditions, which in turn promotes metastasis of EwS cells [13]. Thus, EwS cells can adapt to cellular stress via distinct strategies, which converge in increased invasive properties. We envisage that further elucidating these mechanisms will reveal novel targets for prevention of EwS progression.

\section{Neoangiogenesis \& angiogenic mimicry}

Growing tumors need to maintain blood supply to avoid starvation and hypoxia. EwS accomplish this via three distinct mechanisms.

\section{- Induction of neoangiogenesis}

Apart from the YBX1-mediated induction of HIF1 $\alpha$ [12], EwS can prevent hypoxia through WT1, which is overexpressed in EwS. Under hypoxic conditions, WT1 is further upregulated and stimulates the expression of VEGF [14]. In addition, WT1 regulates the expression of angiopoietin-1 and -2 and its receptor, TIE2, which also promote neoangiogenesis [14]. In accordance, EwS tumors with high levels of WT1 exhibit increased vessel density and are associated with worse patient outcome [14].

Another factor involved in angiogenesis of EwS is the EWSR1-FLI1 target gene CAV1 [15]. CAV1 seems to activate the AKT pathway through EPHA2, thereby causing bFGF production, which promotes endothelial cell migration and angiogenesis [15].

\section{- Modulation of EWSR1-FLI1 levels}

HIF1 $\alpha$ also has another important role, as it appears to be involved in the accumulation of EWSR1-FLI1 protein under hypoxic conditions, which modulates the expression of EWSR1-FLI1 target genes, and is consequently associated with increased invasive growth and a more aggressive metastatic behavior of EwS tumors [16].

\section{- Tumor cell plasticity}

EwS cells exposed to hypoxia form so called 'blood lakes' [17]. Blood lakes are blood-conducting channels that are not lined by endothelial cells [17], but rather by tumor-derived cells, which underwent 
a morphological transformation called 'vasculogenic mimicry' [17]. The mechanisms driving this cellular plasticity remain to be defined. Yet, van der Schaft et al. found that the amount of blood lakes correlates with poor clinical outcome [17], suggesting clinical relevance.

In the future, illuminating the specific ways of how EwS ensures blood supply will provide valuable targets for specifically blocking EwS progression.

\section{Invasion \& metastasis}

Various genes promote EwS invasion and metastasis by different mechanisms [18]. However, for most of them, no specific therapeutic compound is available. Moreover, it seems unlikely that targeting a single gene will prevent EwS metastasis due to extensive signaling crosstalk and network rewiring.

Despite this, many studies share the concept that EwS cells are highly motile and invasive because EwS per se is a highly metastatic cancer entity; recent data suggest that the opposite might be true and that EwS cells utilize a intuitively paradoxical way to disseminate: EwS might not metastasize actively via enhanced cellular migration and invasion, but rather passively through reduced cellular adhesion, which is directly mediated by EWSR1-FLI1 [19]. Indeed, EWSR1-FLI1 expression reduces motility of EwS cells, but at the same time also adhesion by downregulation of important adhesion genes such as Zyxin and integrin $\alpha 5$. EWSR1-FLI1 thereby enables EwS cells to detach passively from the primary tumor and to disseminate via the blood stream [20]. Similarly, EWSR1-FLI1 indirectly downregulates many additional genes involved in cellular adhesion via its direct target gene GLI1 [21]. We anticipate that further research on this 'passive metastasis program' will provide new avenues for specific pharmacologic prevention of EwS progression.

\section{Immunotherapy of Ewing sarcoma}

More than 120 years after the first successful immunotherapy treatment of human sarcoma with streptococcal bacteria by William Coley [22], there is a current renaissance of sarcoma immunotherapy. One of the most promising strategies covers the adoptive transfer of allogeneic NK cells. Cho et al. discovered a profound cytotoxic effect of allogeneic NK cells on EwS cells, which was independent on MHC class I expression [23].

In contrast to NK cells, T cells display a much higher specificity. Accordingly, T cells targeting
EWSR1-FLI1 or its target genes are under intensive investigation. Despite the rather poor immunogenicity of common EwS-associated antigens and native peptides of the EWSR1-FLI1 breakpoint region [24], engineered HLA-A2.1restricted peptides can induce tumor-specific cytotoxic T cells in vitro and kill EwS cells in xenograft models [24].

Lehner et al. combined the advantages of both $\mathrm{NK}$ and $\mathrm{T}$ cells by fusing the extracellular domain of the NKG2D-receptor to an intracellular CD3/CD28 signaling domain enabling redirection of genetically engineered $\mathrm{T}$ cells into the tumor [25]. Finally, EwS-associated antigen-specific allorestricted cytotoxic $\mathrm{T}$ cells have been developed, which enable the use of high-avidity T-cell receptors with reduced risk of graft-versus-host disease [26].

We foresee that immunological approaches based on adoptive transfer of NK or T cells will enter routine clinical practice in the near future. However, we believe that due to the poor immunogenicity of EwS cells, additional immunemodulating strategies, such as the administration of anti-PD-1 antibodies, will be required to ensure a sustained immunological anti-tumor response.

Preventing EwS cells from seeding metastases is a major goal of current EwS research. The aforementioned aspects will continue to be exciting and promising topics and when addressed therapeutically in combination may hold great promise for ultimately halting EwS progression.

\section{Financial \& competing interests disclosure}

$M$ Dallmayer is supported by a scholarship of the 'Deutsche Stiftung für junge Erwachsene mit Krebs'. TGP Grünewald is supported by a grant from the 'Verein zur Förderung von Wissenschaft und Forschung an der Medizinischen Fakultät der LMU München (WiFoMed)', the Daimler and Benz Foundation in cooperation with the Reinhard Frank Foundation, by LMU Munich's Institutional Strategy 'LMUexcellent' within the framework of the German Excellence Initiative, the 'Mehr LEBEN für krebskranke Kinder - Bettina-Bräu-Stiftung', the Walter Schulz Foundation, the Fritz Thyssen Foundation (FTF40.15.0.030MN) and the German Cancer Aid (DKH111886). The authors have no other relevant affiliations or financial involvement with any organization or entity with a financial interest in or financial conflict with the subject matter or materials discussed in the manuscript apart from those disclosed.

No writing assistance was utilized in the production of this manuscript.

\author{
“Preventing Ewing \\ sarcoma cells from seeding \\ metastases is a major goal \\ of current Ewing sarcoma \\ research.”
}




\section{References}

1 Heymann D. Bone Cancer: Primary Bone Cancers and Bone Metastases. Elsevier, CA, USA (2014).

2 Grünewald TGP, Delattre O. Cooperation between somatic mutations and germline susceptibility variants in tumorigenesis - a dangerous liaison. Mol. Cell. Oncol. doi:10.10 80/23723556.2015.1086853 (2015) (Epub ahead of print).

3 Sand LGL, Szuhai K, Hogendoorn PCW. Sequencing overview of Ewing sarcoma: a journey across genomic, epigenomic and transcriptomic landscapes. Int. J. Mol. Sci. 16(7), 16176-16215 (2015).

4 Grünewald TGP, Bernard V, GilardiHebenstreit P et al. Chimeric EWSR1-FLI1 regulates the Ewing sarcoma susceptibility gene EGR2 via a GGAA-microsatellite. Nat. Genet. 47(9), 1073-1078 (2015).

5 Gangwal K, Lessnick SL. Microsatellites are EWS/FLI response elements: genomic "junk" is EWS/FLI's treasure. Cell Cycle 7(20), 3127-3132 (2008).

6 Riggi N, Knoechel B, Gillespie SM et al. EWS-FLI1 utilizes divergent chromatin remodeling mechanisms to directly activate or repress enhancer elements in Ewing sarcoma. Cancer Cell 26(5), 668-681 (2014).

7 Luo W, Gangwal K, Sankar S, Boucher KM, Thomas D, Lessnick SL. GSTM4 is a microsatellite-containing EWS/FLI target involved in Ewing's sarcoma oncogenesis and therapeutic resistance. Oncogene 28(46), 4126-4132 (2009).

8 García-Aragoncillo E, Carrillo J, Lalli E et al. DAX1, a direct target of EWS/FLI1 oncoprotein, is a principal regulator of cell-cycle progression in Ewing's tumor cells. Oncogene 27(46), 6034-6043 (2008).

9 Kwon I, Kato M, Xiang S et al. Phosphorylation-regulated binding of RNA polymerase II to fibrous polymers of low-complexity domains. Cell 155(5), 1049-1060 (2013).

10 Monument MJ, Johnson KM, McIlvaine E et al. Clinical and biochemical function of polymorphic NR0B1 GGAA-microsatellites in Ewing sarcoma: a report from the Children's Oncology Group. PLoS ONE 9(8), e104378 (2014).

11 Somasekharan SP, El-Naggar A, Leprivier G et al. YB-1 regulates stress granule formation and tumor progression by translationally activating G3BP1. J. Cell Biol. 208(7), 913-929 (2015).

12 El-Naggar AM, Veinotte CJ, Cheng H et al. Translational activation of HIF1 $\alpha$ by YB-1 promotes sarcoma metastasis. Cancer Cell 27(5), 682-697 (2015).

13 Krook MA, Nicholls LA, Scannell CA, Chugh R, Thomas DG, Lawlor ER. Stress-induced CXCR4 promotes migration and invasion of Ewing sarcoma. Mol. Cancer Res. 12(6), 953-964 (2014).

14 Katuri V, Gerber S, Qiu X et al. WT1 regulates angiogenesis in Ewing sarcoma. Oncotarget 5(9), 2436-2449 (2014).

15 Sáinz-Jaspeado M, Huertas-Martinez J, Lagares-Tena L et al. EphA2-induced angiogenesis in ewing sarcoma cells works through bFGF production and is dependent on caveolin-1. PLoS ONE 8(8), e71449 (2013).

16 Aryee DNT, Niedan S, Kauer M et al. Hypoxia modulates EWS-FLI1 transcriptional signature and enhances the malignant properties of Ewing's sarcoma cells in vitro. Cancer Res. 70 (10), 4015-4023 (2010).

17 van der Schaft DWJ, Hillen F, Pauwels P et al. Tumor cell plasticity in Ewing sarcoma, an alternative circulatory system stimulated by hypoxia. Cancer Res. 65(24), 11520-11528 (2005).

18 Mackintosh C, Madoz-Gúrpide J, Ordóñez JL, Osuna D, Herrero-Martín D. The molecular pathogenesis of Ewing's sarcoma. Cancer Biol. Ther. 9(9), 655-667 (2010).

19 Chaturvedi A, Hoffman LM, Welm AL, Lessnick SL, Beckerle MC. The EWS/FLI oncogene drives changes in cellular morphology, adhesion, and migration in Ewing sarcoma. Genes Cancer 3(2), 102-116 (2012).

20 Chaturvedi A, Hoffman LM, Jensen CC et al. Molecular dissection of the mechanism by which EWS/FLI expression compromises actin cytoskeletal integrity and cell adhesion in Ewing sarcoma. Mol. Biol. Cell. 25(18), 2695-2709 (2014).

21 Sankar S, Tanner JM, Bell R et al. A novel role for keratin 17 in coordinating oncogenic transformation and cellular adhesion in Ewing sarcoma. Mol. Cell. Biol. 33(22), 4448-4460 (2013).

22 Coley WB. II. Contribution to the knowledge of sarcoma. Ann. Surg. 14(3), 199-220 (1891).

23 Cho D, Shook DR, Shimasaki N, Chang Y-H, Fujisaki H, Campana D. Cytotoxicity of activated natural killer cells against pediatric solid tumors. Clin. Cancer Res. 16(15), 3901-3909 (2010).

24 Evans CH, Liu F, Porter RM et al. EWS-FLI1-targeted cytotoxic T-cell killing of multiple tumor types belonging to the Ewing sarcoma family of tumors. Clin. Cancer Res. 18(19), 5341-5351 (2012).

25 Lehner M, Götz G, Proff J et al. Redirecting T cells to Ewing's sarcoma family of tumors by a chimeric NKG2D receptor expressed by lentiviral transduction or mRNA transfection. PLoS ONE 7(2), e31210 (2012).

26 Thiel U, Pirson S, Müller-Spahn C et al. Specific recognition and inhibition of Ewing tumour growth by antigen-specific allorestricted cytotoxic T cells. Br. J. Cancer 104(6), 948-956 (2011). 